\title{
Voltage Gated Potassium Channel Antibody Encephalitis Associated with
} Hyperglycemia

\author{
Benjamin R Kummer ${ }^{1 *}$, Bethany L Johnson Kerner ${ }^{2}$ and Charles C Esenwa ${ }^{1}$ \\ ${ }^{1}$ Department of Neurology, Columbia University Medical Center, New York, NY, USA \\ ${ }^{2}$ College of Physicians and Surgeons, Columbia University Medical Center, New York, NY, USA
}

\begin{abstract}
A 65-year-old man presented for evaluation of altered mental status, preceded by several months of sleep abnormalities, excessive salivation, and paroxysms of drenching sweats. Despite having no history of diabetes mellitus, he was severely hyperglycemic with a hemoglobin A1C of $11 \%$. Serum tests revealed hyponatremia as well as positive Voltage-Gated Potassium Channel (VGKC) serum antibodies, and EEG showed frequent epileptic discharges originating from the left temporal lobe. He was ultimately diagnosed with VGKC antibody encephalitis and was treated with antiepileptic, anti-diabetic and corticosteroid agents with improvement in his mental status. One year following treatment, he was seizure-free, had returned to his baseline level of function, and had been weaned off both corticosteroids and anti-diabetic medications.
\end{abstract}

Keywords: Mental status; Voltage gated potassium channel; Syndrome of inappropriate anti-diuretic hormone secretion

\section{Case Presentation}

In February 2013, a 65-year-old man with well-controlled hypertension and low testosterone presented to the hospital for evaluation of multiple, progressive, neurologic complaints. In 2012, he had begun to experience fragmented sleep, as well as intermittent episodes of drenching sweats, with hot flashes during the day and hypersalivation at night. In January 2013, he developed paroxysmal episodes of self-limited confusion and periods of unexplained shortterm memory loss. In early February, he became aware of two weeks of polyuria and polydipsia. The patient had had regular follow up with his endocrinologist for management of low testosterone levels and was serially screened for diabetes mellitus; the most recent serum glucose test had been in late December 2012 and had not been significantly elevated. Collateral history from his endocrinologist and primary care doctor confirmed that he had had no history of diabetes or impaired glucose tolerance. He did not have a family history of autoimmune disease and was a high-functioning executive, working as recently as two weeks prior to presentation. He drank alcohol socially and denied any further toxic habits.

Initial examination at presentation to hospital in February 2013 revealed sinus tachycardia to $140 \mathrm{bpm}$. He appeared calm and well groomed. He initially had a normal level of consciousness, attention and language function, interceded by brief episodes of sudden verbal unresponsiveness accompanied by rhythmic right arm shaking and followed by minutes of lethargy. Although his immediate recall was normal during his lucid intervals, he was unable to remember events from several days prior. The rest of his neurological examination was unremarkable. His mental status waxed and waned throughout his hospital stay, alternating between periods of wakefulness, lethargy and occasional stupor.

Initial serum glucose at presentation was $540 \mathrm{mg} / \mathrm{dL}$ (reference range $75-100 \mathrm{mg} / \mathrm{dL}$ ). Serum sodium was $127 \mathrm{mg} / \mathrm{dL}$ (reference range $136-146 \mathrm{mg} / \mathrm{dL}$ ) with urine electrolytes consistent with the Syndrome of Inappropriate Anti-Diuretic Hormone Secretion (SIADH). Hemoglobin A1C was significantly elevated to $11 \%$ (reference limit of normal $<6.5 \%$ ). Ferritin level was markedly elevated at $1339 \mathrm{mg} / \mathrm{dl}$ (reference range 29-248 mg/dL). Anti-Glutamic Acid Decarboxylase (GAD) antibody test was negative. CSF profile from February 2013 showed normal glucose and protein with a white blood cell count of 3/ $\mu \mathrm{L}$ and red blood cell count of $269 / \mu \mathrm{L}$ (reference range $0-5$ and 0 cells/ $\mu \mathrm{L}$, respectively). CSF herpes simplex virus 1 and 2 PCRs, in addition to other viral PCRs (VZV, EBV, and CMV) were negative. Serum TSH, prolactin, growth hormone, and C-peptide were normal. Transferrin saturation was $18 \%$; levels greater than $16 \%$ are considered normal; those above $45 \%$ are typically associated with iron overload [1]. Serum quantitative voltage-gated potassium channel antibody levels (Mayo Medical Laboratories, determined via radio-immunoprecipitation) were $0.07 \mathrm{nmol} / \mathrm{mL}$ (reference range $<0.02 \mathrm{nmol} / \mathrm{mL}$; other authors have proposed $<0.1 \mathrm{nmol} / \mathrm{L}$ ) [2]

Video Electroencephalographic (vEEG) investigation in early February 2013 revealed frequent left temporal lobe electrographic seizures, correlating to periods of somnolence with subtle rhythmic movements of the right hand. Magnetic resonance imaging (MRI) of the brain with and without contrast revealed no abnormal enhancement, with mild hyperintensity seen on fluid-attenuated inversion recovery (FLAIR) sequences in the medial temporal lobes bilaterally (Figure 1). Whole-body computed tomography and eventual Positron Emission Tomography (PET) were negative for malignancy. Abdominal ultrasound revealed hepatic steatosis without evidence of cirrhosis, and the patient's low-normal transferrin saturation level effectively ruled out iron overload.

The patient's fluctuating levels of consciousness and seizures, along with a lack of clearly lateralizing neurologic examination findings, suggest a diffuse process affecting the central nervous system. As such, the differential diagnosis is wide. New-onset diabetes resulting in a hyper-osmotic non-ketotic state with osmotic diuresis and hypovolemic hyponatremia is an immediate possibility. Inflammatory or vascular conditions, such as neurological sarcoidosis or cerebral vasculitis are also possible, especially considering the patient's elevated

*Corresponding author: Benjamin R Kummer, Department of Neurology, Columbia University Medical Center, 710 W 168th Street, 14th Floor, New York, NY, 10032 USA, E-mail: brk2104@cumc.columbia.edu

Received November 12, 2014; Accepted December 28, 2014; Published December 31, 2014

Citation: Kummer BR, Johnson Kerner BL, Esenwa CC (2014) Voltage Gated Potassium Channel Antibody Encephalitis Associated with Hyperglycemia. J Clin Case Rep 4: 464. doi:10.4172/2165-7920.1000464

Copyright: (c) 2014 Kummer BR, et al. This is an open-access article distributed under the terms of the Creative Commons Attribution License, which permits unrestricted use, distribution, and reproduction in any medium, provided the original author and source are credited. 


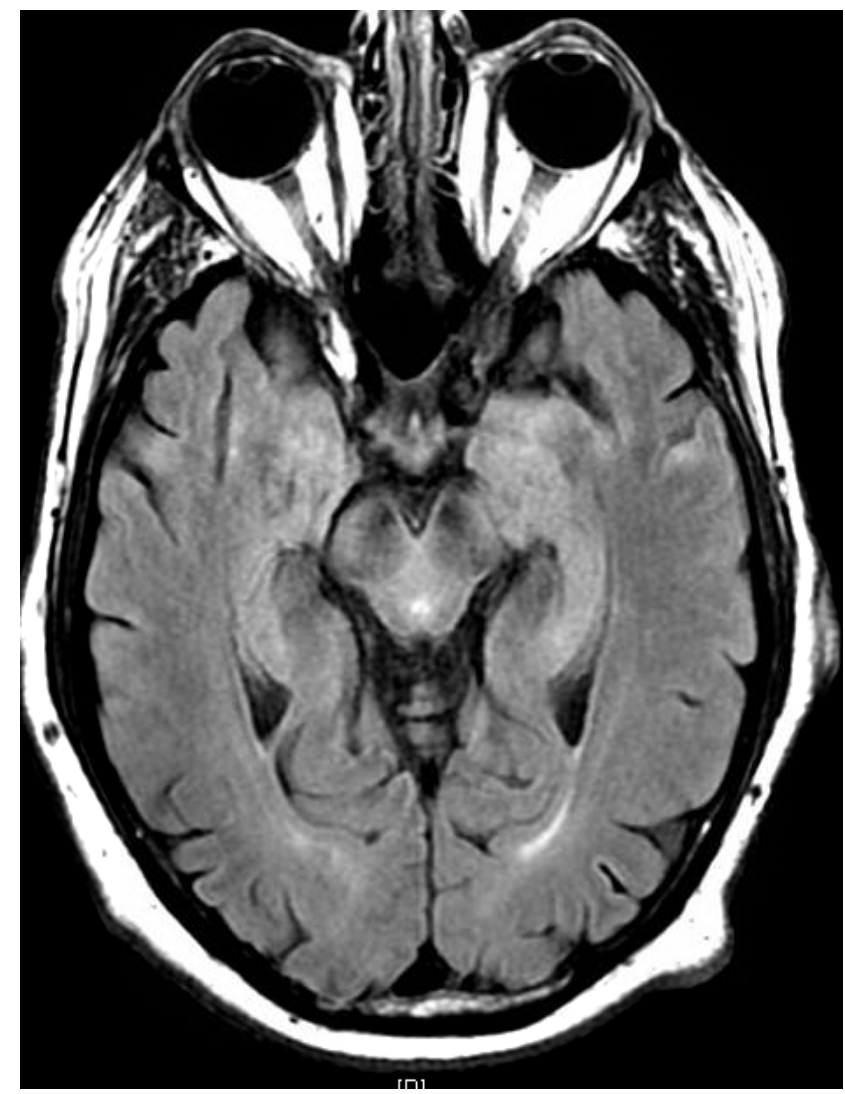

Figure 1: Fluid-attenuation inversion recovery (FLAIR) MRI of the brain, showing mild bilateral hyperintense signal in the medial temporal lobes.

ferritin, which is typically high in chronic inflammation. Infectious etiologies, such as chronic viral or Borrelia burgdorferi encephalitis, are also possible in this case. Finally, given the patient's ongoing seizures, the possibility of primary epileptic encephalopathy from repeated seizures must also be considered. Taken together, the patient's months of preceding neurological symptoms, combined with seizures, metabolic disturbances, serum testing and suggestive MRI findings are most consistent with a diagnosis of voltage-gated potassium channel antibody encephalitis.

The patient was treated with insulin for hyperglycemia, and levetiracetam for seizures. Despite normalization of the patient's serum glucose levels and treatment with anti-epileptic medication, subsequent EEGs recorded several days after the initial discovery of the patient's seizures continued to show epileptic activity in the left temporal lobe, ultimately requiring the addition of both phenytoin and pregabalin to achieve electrographic seizure freedom.

The patient's mental status improved only after his electrographic seizures ceased. He was discharged home in late February 2013 on a combination of metformin, levetiracetam, phenytoin, pregabalin, and prednisone. However, he continued to have mild short-term memory impairment. He was re-admitted in March 2013 for an elective trial of high dose methylprednisolone, whereupon he experienced improvement in his cognition (Folstein Mini Mental Status Exam scores were 28 and 30, before and after methylprednisolone, respectively). His follow-up hemoglobin A1C, while still on steroids, was $6.7 \%$ in late March 2013. Prednisone was continued as a slow taper over the course of approximately 1 year. Levetiracetam, and metformin were also tapered off by May 2014. VGKC antibody level at this time was 0.00 $\mathrm{nmol} / \mathrm{L}$, and the patient was noted to have returned to his prior level of functioning. However, he remained on both pregabalin and phenytoin, albeit at lower doses than when he was hospitalized. He was unable to remember most of his first hospitalization.

\section{Discussion}

Voltage Gated Potassium Channel (VGKC) antibody-associated encephalitis is an autoimmune limbic encephalitis caused by binding of antibodies to voltage-gated potassium channel associated proteins. VGKC antibodies were first discovered by Shillito et al. [3]. These antibodies were present in three of six patients with neuromyotonia, a syndrome of peripheral nervous system hyper-excitability characterized by myokymia, cramps, impaired muscle relaxation, sporadic muscle weakness and elevated serum levels of creatine kinase. The constellation of signs and symptoms outlined by Shillito in 1995 overlaps with a syndrome of insomnia, confusion, hallucinations, neuromyotonia and autonomic hyper-excitability described in two patients by Morvan in 1890 [4]. This very syndrome, when combined with the presence of VGKC antibodies, is now referred to as Morvan syndrome.

In 2001, Buckley et al. described two women with positive VGKC antibodies who presented with peripheral hyper-excitability and cognitive dysfunction. This was the first report to associate limbic encephalitis with VGKC-associated antibodies [5]. Since then, the clinical presentation of central nervous system involvement has expanded to include acute onset seizures [6] and rapidly progressive dementia [7]. It is now thought that the VGKC-associated syndromes exist on a spectrum that contains both peripheral and central nervous system dysfunction, ranging from limbic encephalitis to neuromyotonia and the Morvan syndrome itself [8]. VGKC encephalitis is believed to be the most common of the immune-mediated encephalitides. It is more commonly found in men, with a mean presenting age of 65 [9]. In contrast to classical limbic encephalitis, which is typically thought of as paraneoplastic, VGKC-antibody associated limbic encephalitis is usually idiopathic [6]. It has also been found in association with thymoma as well as lung and testicular tumors [5].

Recent research has shown that specific clinical syndromes can be traced to autoimmune activity targeted against potassium channelassociated proteins rather than the channel complex itself. In a 57-patient case series of patients with clinical limbic encephalitis and positive anti-potassium channel antibodies, Lai et al. demonstrated that the antibodies previously thought to attack the potassium channel complex actually target the channel-associated proteins LG1 or CASPR2. A large proportion of the patients in this series had the LG1 subtype and had symptoms of classical limbic encephalitis: $60 \%$ presented with hyponatremia, $82 \%$ presented with clinical seizures, and all patients presented with memory loss [10]. The CASPR2 subtype, on the other hand, is often responsible for the peripheral variants of this syndrome. More recently, a variant with antibodies to dipeptidyl-peptidase-like protein-6 (DPPX), a cell surface auxiliary unit of the Kv4.2 potassium channel, was reported in four patients with subacute central nervous system hyperexcitability (agitation, myoclonus, tremor and seizures) neuropsychiatric and prominent gastrointestinal symptoms [11]. There was a robust expression of this protein in the myenteric plexus, providing an explanation for the associated gastrointestinal symptoms. This type of cross-reactivity is a well-described aspect of other antibody mediated diseases like Sjögren's disease, rheumatoid arthritis and the spectrum of anti-GAD disorders, which all have variable multi-organ manifestations. 
Hyponatremia is a relatively common presenting finding of VGKC antibody encephalitis, occurring in $36 \%$ of cases described in a 72-patient meta-analysis [12]. The mechanism has not yet been delineated, but pituitary and hypothalamic dysfunction have been proposed [10]. Hyperglycemia, however, is not a recognized feature of this syndrome, and to date, only one case of VGKC encephalitis has been reported where hyperglycemia was noted at presentation. In this case report, a 51-year-old man presented with a fluctuating epileptic encephalopathy and hyperglycemia to $463 \mathrm{mg} / \mathrm{dL}$. Following treatment with glucocorticoids for one year, the patient returned to his premorbid level of functioning, though glucose levels at follow-up were not reported [13]. Although diabetes mellitus type II is fairly common, the presentation of sudden-onset, florid hyperglycemia without a previous diagnosis of diabetes, as in our case, is very unusual. If a link between glucose levels and VGKC antibodies does exist, we offer two potential explanations: direct antibody effects on the pancreas, or hypothalamic/ pituitary dysregulation.

Voltage Gated Potassium Channels (VGKC) are expressed in many tissues throughout the body, including the liver and skeletal muscle. In animal models, VGKC have been linked to insulin sensitivity and glucose tolerance [14]. In humans, a single-nucleotide polymorphism (T-1645C allele) in the promoter region of the Kv1.3 potassium channel protein is associated with higher plasma glucose concentrations and lower insulin sensitivity [15]. In addition, common mutations in KCNQ1, a gene which encodes subunits of the Kv7.1 potassium channel in Japanese and Dutch populations, are associated with type 2 diabetes, which can be attributed in part to an effect on insulin secretion [16]. Auto-antibodies against VGKC pore complex-associated proteins may similarly affect the insulin secretion indirectly by affecting the function of potassium channels in the pancreas. Our patient had a normal C-peptide level, arguing against disruption of insulin production (typically seen in diabetes mellitus type 1). Antibody-mediated dysregulation of hypothalamic Corticotropin-Releasing Hormone (CRH) secretion could theoretically cause disruption of ACTH and cortisol secretion, thus leading to a Cushing-type pathophysiology. A case-control experiment examining levels of cortisol and IGF-1 in patients with VGKC antibody encephalitis over the course of the disease may be a helpful study design to shed light on this mechanistic possibility.

This particular case raises a number of important points, most notably the possibility that the patient developed diabetes mellitus in the month preceding his admission to the hospital. Several facts argue against this. The patient's hemoglobin A1c of $11 \%$ at presentation, despite normoglycemia one month prior, suggests a rapid and sustained development of severely elevated blood glucose levels after his most recent screen, i.e. over four weeks or less. Average blood glucose levels can be reliably extrapolated for any given hemoglobin A1c value [17], but this relationship was originally described in patients without large variations in serum glucose. Also, the hemoglobin Alc is theoretically calculated as a weighted average of glycated hemoglobin in a given blood sample, and thus more heavily reflects the glycation of recently produced circulating red blood cells [18]. It is therefore altogether possible that severe, continuous hyperglycemia over a relatively short period of time - caused by an autoimmune process rather than diabetes - could have resulted in a hemoglobin Alc value of $11 \%$. More importantly, the patient's requirements for anti-diabetic medications ceased at the conclusion of his immunomodulatory treatment with prednisone. The patient had returned to a normal state of mental functioning at this point as well, thus strengthening the link between hyperglycemia and the presence of encephalitis.
The VGKC antibody levels of zero at follow-up are likely due to chronic effects of glucocorticoids on the immune system. Corticosteroids primarily affect cell-mediated immunity more than humoral immunity, but have been described to inhibit immunoglobulin production particularly if used in the long-term [19]. It is likely that the effect of long-term steroids on antibody production, in combination with decreased antigen presentation by $\mathrm{T}$ helper cells, produced such a low level of antibody one year after treatment. An additional important point to note is that despite positive serum VGKC antibodies, further testing for antibodies against potassium channel complex subunits or associated proteins was not performed. Subunit testing might allow further understanding of the relationship between different populations of voltage-gated potassium channels and glucose regulation.

Our report is unique in that it illustrates the neurological syndrome of VGKC antibody-mediated encephalitis preceding the development of hyperglycemia. The patient described had had prior normal blood glucose testing, limiting the possibility of glucose metabolism dysfunction during his several months of neurological symptoms leading up to his hospitalization. We propose a pathophysiologic mechanism that could explain the association between this antibodymediated neurological syndrome and impaired blood glucose metabolism. It may be prudent to recognize similar presentations as our patients' as a result of an autoimmune process causing a patient's encephalopathy, rather than presuming that metabolic derangement itself is the underlying etiology. Recognizing and further describing these metabolic derangements found in association with neuronal autoantibodies may add a new dimension to the evolving and intersecting areas of neurology, immunology and molecular biology.

\section{References}

1. CDC Hemochromatosis (Iron Storage Disease) Training Diagnostic Testing Protocol.

2. Vincent A, Bien CG, Irani SR, Waters P (2011) Autoantibodies associated with diseases of the CNS: new developments and future challenges. Lancet Neurol 10: 759-772.

3. Shillito P, Molenaar PC, Vincent A, Leys K, Zheng W, et al. (1995) Acquired neuromyotonia: evidence for autoantibodies directed against $\mathrm{K}+$ channels of peripheral nerves. Ann Neurol 38: 714-722.

4. Morvan, A (1890) De la chorée fibrillaire. Gazette Hébdomadaire de Médecine et de Chirurgie. 27, 173-200.

5. Buckley C, Oger J, Clover L, Tüzün E, Carpenter K, et al. (2001) Potassium channel antibodies in two patients with reversible limbic encephalitis. Ann Neurol 50: 73-78.

6. Vincent A, Buckley C, Schott JM, Baker I, Dewar BK, et al. (2004) Potassium channel antibody associated encephalopathy: a potentially immunotherapyresponsive form of limbic encephalitis. Brain: a journal of neurology. 127: 701 712

7. Geschwind MD, Shu H, Haman A, Sejvar JJ, Miller BL (2008) Rapidly progressive dementia. Ann Neurol 64: 97-108.

8. Kleopa KA, Elman LB, Lang B, Vincent A, Scherer SS (2006) Neuromyotonia and limbic encephalitis sera target mature Shaker-type $\mathrm{K}+$ channels: subunit specificity correlates with clinical manifestations. Brain: a journal of neurology 129 1570-1584.

9. Buckley C (2005) Diseases Associated with Antibodies to Voltage-Gated Potassium Channels. Advances in Clinical Neuroscience and Rehabilitation. 5: 11-12.

10. Lai M, Huijbers MG, Lancaster E, Graus F, Bataller L, et al. (2010) Investigation of LGI1 as the antigen in limbic encephalitis previously attributed to potassium channels: a case series. Lancet Neurology. 9: 776-785.

11. Boronat A, Gelfand JM, Gresa-Arribas N, Jeong HY, Walsh M, et al. (2013) Encephalitis and antibodies to dipeptidyl-peptidase-like protein-6, a subunit of Kv4.2 potassium channels. Ann Neurol 73: 120-128. 
Citation: Kummer BR, Johnson Kerner BL, Esenwa CC (2014) Voltage Gated Potassium Channel Antibody Encephalitis Associated with Hyperglycemia. J Clin Case Rep 4: 464. doi:10.4172/2165-7920.1000464

12. Tan KM, Lennon VA, Klein CJ, Boeve BF, Pittock SJ (2008) Clinical spectrum of voltage-gated potassium channel autoimmunity. Neurology 70: 1883-1890.

13. Scott JT, Scally C, Peden N, Macleod M (2012) Voltage-gated potassium channel antibody-related encephalopathy: a case which may extend the documented phenotype of this condition. BMJ Case Reports.

14. Xu J, Wang P, Li Y, Li G, Kaczmarek LK, et al. (2004) The voltage-gated potassium channel Kv1.3 regulates peripheral insulin sensitivity. Proc Natl Acad Sci U S A 101: 3112-3117.

15. Tschritter O, Machicao F, Stefan N, Schäfer S, Weigert C, et al. (2006) A new variant in the human Kv1.3 gene is associated with low insulin sensitivity and impaired glucose tolerance. J Clin Endocrinol Metab 91: 654-658.
16. van Vliet-Ostaptchouk JV, van Haeften TW, Landman GW, Reiling E, Kleefstra $\mathrm{N}$, et al. (2012) Common variants in the type 2 diabetes KCNQ1 gene are associated with impairments in insulin secretion during hyperglycaemic glucose clamp. PLoS One 7: e32148.

17. Nathan DM, Kuenen J, Borg R, Zheng H, Schoenfeld D, et al. (2008) Translating the $\mathrm{A} 1 \mathrm{C}$ assay into estimated average glucose values. Diabetes Care 31:14731478.

18. National Glycohemoglobin Standardization Program (NGSP) website: HbA1c and Estimated Average Glucose (eAG)

19. Fedor ME, Rubinstein A (2006) Effects of long-term low-dose corticosteroid therapy on humoral immunity. Ann Allergy Asthma Immunol 97: 113-116. 